\title{
Descent polynomials for permutations with bounded drop size
}

\author{
Fan Chung ${ }^{1}$, Anders Claesson ${ }^{2}$, Mark Dukes $\|^{3}$ and Ronald Graham ${ }^{1}$ \\ ${ }^{1}$ University of California at San Diego, La Jolla CA 92093, USA. \\ ${ }^{2}$ The Mathematics Institute, School of Computer Science, Reykjavík University, 103 Reykjavík, Iceland. \\ ${ }^{3}$ Science Institute, University of Iceland, 107 Reykjavík, Iceland.
}

\begin{abstract}
Motivated by juggling sequences and bubble sort, we examine permutations on the set $\{1,2, \ldots, n\}$ with $d$ descents and maximum drop size $k$. We give explicit formulas for enumerating such permutations for given integers $k$ and $d$. We also derive the related generating functions and prove unimodality and symmetry of the coefficients.

Résumé. Motivés par les "suites de jonglerie” et le tri à bulles, nous étudions les permutations de l'ensemble $\{1,2, \ldots, n\}$ ayant $d$ descentes et une taille de déficience maximale $k$. Nous donnons des formules explicites pour l'énumération de telles permutations pour des entiers $k$ et $d$ fixés, ainsi que les fonctions génératrices connexes. Nous montrons aussi que les coefficients possèdent des propriétés d'unimodalité et de symétrie.
\end{abstract}

Keywords: Permutations, descent polynomial, drop size, Eulerian distribution.

\section{Introduction}

There have been extensive studies of various statistics on $\mathcal{S}_{n}$, the set of all permutations of $\{1,2, \ldots, n\}$. For a permutation $\pi$ in $\mathcal{S}_{n}$, we say that $\pi$ has a drop at $i$ if $\pi_{i}<i$ and that the drop size is $i-\pi_{i}$. We say that $\pi$ has a descent at $i$ if $\pi_{i}>\pi_{i+1}$. One of the earliest results [8] in permutation statistics states that the number of permutations in $\mathcal{S}_{n}$ with $k$ drops equals the number of permutations with $k$ descents. A concept closely related to drops is that of excedances, which is just a drop of the inverse permutation. In this paper we focus on drops instead of excedances because of their connection with our motivating applications concerning bubble sort and juggling sequences.

Other statistics on a permutation $\pi$ include such things as the number of inversions (pairs $(i, j)$ such that $i<j$ and $\pi_{i}>\pi_{j}$ ) and the major index of $\pi$ (the sum of $i$ for which a descent occurs). The enumeration of and generating functions for these statistics can be traced back to the work of Rodrigues in 1839 [9] but was mainly influenced by McMahon's treatise in 1915 [8]. There is an extensive literature studying the distribution of the above statistics and their $q$-analogs, see for example Foata and Han [4], or the papers of Shareshian and Wachs [10, 11] for more recent developments.

This joint work originated from its connection with a paper [2] on sequences that can be translated into juggling patterns. The set of juggling sequences of period $n$ containing a specific state, called the ground

\footnotetext{
${ }^{\dagger} \mathrm{AC}$ and MD were supported by grant no. 090038011 from the Icelandic Research Fund. 
state, corresponds to the set $\mathcal{B}_{n, k}$ of permutations in $\mathcal{S}_{n}$ with drops of size at most $k$. As it turns out, $\mathcal{B}_{n, k}$ can also be associated with the set of permutations that can be sorted by $k$ operations of bubble sort. These connections will be further described in the next section. We note that the maxdrop statistic has not been treated in the literature as extensively as many other statistics in permutations. As far as we know, this is the first time that the distribution of descents with respect to maxdrop has been determined.

First we give some definitions concerning the statistics and polynomials that we examine. Given a permutation $\pi$ in $\mathcal{S}_{n}$, let $\operatorname{Des}(\pi)=\left\{1 \leq i<n: \pi_{i}>\pi_{i+1}\right\}$ be the descent set of $\pi$ and let $\operatorname{des}(\pi)=$ $|\operatorname{Des}(\pi)|$ be the number of descents. We use $\operatorname{maxdrop}(\pi)$ to denote the value of the maximum drop (or maxdrop) of $\pi$,

$$
\operatorname{maxdrop}(\pi)=\max \{i-\pi(i): 1 \leq i \leq n\} .
$$

Let $\mathcal{B}_{n, k}=\left\{\pi \in \mathcal{S}_{n}: \operatorname{maxdrop}(\pi) \leq k\right\}$. It is known, and also easy to show, that $\left|\mathcal{B}_{n, k}\right|=k !(k+1)^{n-k}$; e.g., see [2, Thm. 1] or [7, p. 108]. Let

$$
b_{n, k}(r)=\left|\left\{\pi \in \mathcal{B}_{n, k}: \operatorname{des}(\pi)=r\right\}\right|,
$$

and define the ( $k$-maxdrop-restricted) descent polynomial

$$
B_{n, k}(x)=\sum_{r \geq 0} b_{n, k}(r) x^{r}=\sum_{\pi \in \mathcal{B}_{n, k}} x^{\operatorname{des}(\pi)} .
$$

Examining the case of $k=2$, we observed the coefficients $b_{n, 2}(r)$ of $B_{n, 2}(x)$ appear to be given by every third coefficient of the simple polynomial

$$
\left(1+x^{2}\right)\left(1+x+x^{2}\right)^{n-1} .
$$

Looking at the next two cases, $k=3$ and $k=4$, yielded more mysterious polynomials: $b_{n, 3}(r)$ appeared to be every fourth coefficient of

$$
\left(1+x^{2}+2 x^{3}+x^{4}+x^{6}\right)\left(1+x+x^{2}+x^{3}\right)^{n-2}
$$

and $b_{n, 4}(r)$ every fifth coefficient of

$$
\left(1+x^{2}+2 x^{3}+4 x^{4}+4 x^{5}+4 x^{7}+4 x^{8}+2 x^{9}+x^{10}+x^{12}\right)\left(1+x+x^{2}+x^{3}+x^{4}\right)^{n-3} .
$$

After a fierce battle with these polynomials, we were able to show that $b_{n, k}(r)$ is the coefficient of $u^{r(k+1)}$ in the polynomial

$$
P_{k}(u)\left(1+u+\cdots+u^{k}\right)^{n-k}
$$

where

$$
P_{k}(u)=\sum_{j=0}^{k} A_{k-j}\left(u^{k+1}\right)\left(u^{k+1}-1\right)^{j} \sum_{i=j}^{k}\left(\begin{array}{l}
i \\
j
\end{array}\right) u^{-i},
$$

and $A_{k}$ denotes the $k$ th Eulerian polynomial (defined in the next section). Further to this, we give an expression for the generating function $\mathbf{B}_{k}(z, y)=\sum_{n \geq 0} B_{n, k}(y) z^{n}$, namely

$$
\mathbf{B}_{k}(z, y)=\frac{1+\sum_{t=1}^{k}\left(A_{t}(y)-\sum_{i=1}^{t}\left(\begin{array}{c}
k+1 \\
i
\end{array}\right)(y-1)^{i-1} A_{t-i}(y)\right) z^{t}}{1-\sum_{i=1}^{k+1}\left(\begin{array}{c}
k+1 \\
i
\end{array}\right) z^{i}(y-1)^{i-1}} .
$$


We also give some alternative formulations for $P_{k}$ which lead to some identities involving Eulerian numbers as well as proving the symmetry and unimodality of the polynomials $B_{n, k}(x)$.

Many questions remain. For example, is there a more natural bijective proof for the formulas that we have derived for $B_{n, k}$ and $\mathbf{B}_{k}$ ? Why do permutations that are $k$-bubble sortable define the aforementioned juggling sequences?

\section{Descent polynomials, bubble sort and juggling sequences}

We first state some standard notation. The polynomial

$$
A_{n}(x)=\sum_{\pi \in \mathcal{S}_{n}} x^{\operatorname{des}(\pi)}
$$

is called the $n$th Eulerian polynomial. For instance, $A_{0}(x)=A_{1}(x)=1$ and $A_{2}(x)=1+x$. Note that $B_{n, k}(x)=A_{n}(x)$ for $k \geq n-1$, since $\operatorname{maxdrop}(\pi) \leq n-1$ for all $\pi \in \mathcal{S}_{n}$. The coefficient of $x^{k}$ in $A_{n}(x)$ is denoted $\left\langle\begin{array}{l}n \\ k\end{array}\right\rangle$ and is called an Eulerian number. It is well known that [5]

$$
\frac{1-w}{e^{(w-1) z}-w}=\sum_{k, n \geq 0}\left\langle\begin{array}{l}
n \\
k
\end{array}\right\rangle w^{k} \frac{z^{n}}{n !} .
$$

The Eulerian numbers are also known to be given explicitly as [3, 5] $\left\langle\begin{array}{l}n \\ k\end{array}\right\rangle=\sum_{i=0}^{n}\left(\begin{array}{c}n+1 \\ i\end{array}\right)(k+1-i)^{n}(-1)^{i}$.

We define the operator bubble which acts recursively on permutations via

$$
\text { bubble }(L n R)=\operatorname{bubble}(L) R n \text {. }
$$

In other words, to apply bubble to a permutation $\pi$ in $\mathcal{S}_{n}$, we split $\pi$ into (possibly empty) blocks $L$ and $R$ to the left and right, respectively, of the largest element of $\pi$ (which initially is $n$ ), interchange $n$ and $R$, and then recursively apply this procedure to $L$. We will use the convention that bubble $(\emptyset)=\emptyset$; here $\emptyset$ denotes the empty permutation. This operator corresponds to one pass of the classical bubble sort operation. Several interesting results on the analysis of bubble sort can be found in Knuth [7] pp. 106-110]. We define the bubble sort complexity of $\pi$ as

$$
\operatorname{bsc}(\pi)=\min \left\{k: \operatorname{bubble}^{k}(\pi)=\mathrm{id}\right\}
$$

the number of times bubble must be applied to $\pi$ to give the identity permutation. The following lemma is easy to prove using induction.

Lemma 1 (i) For all permutations $\pi$ we have $\operatorname{maxdrop}(\pi)=\operatorname{bsc}(\pi)$.

(ii) The bubble sort operator maps $\mathcal{B}_{n, k}$ to $\mathcal{B}_{n, k-1}$.

The class of permutations $\mathcal{B}_{n, k}$ appears in a recent paper [2] on enumerating juggling patterns that are usually called siteswaps by (mathematically inclined) jugglers. Suppose a juggler throws a ball at time $i$ so that the ball will be in the air for a time $t_{i}$ before landing at time $t_{i}+i$. Instead of an infinite sequence, we will consider periodic patterns, denoted by $T=\left(t_{1}, t_{2}, \ldots, t_{n}\right)$. A juggling sequence is just one in which two balls never land at the same time. It is not hard to show [1] that a necessary and sufficient condition for a sequence to be a juggling sequence is that all the values $t_{i}+i(\bmod n)$ are distinct. In 
particular, it follows that that the average of $t_{i}$ is just the numbers of balls being juggled. Here is an example:

If $T=(3,5,0,2,0)$ then at time 1 a ball is thrown that will land at time $1+3=4$. At time $2 \mathrm{a}$ ball is thrown that will land at time $2+5=7$. At time 3 a ball is thrown that will land at time $3+0=3$. Alternatively one can say that no ball is thrown at time 3 . This is represented in the following diagram.

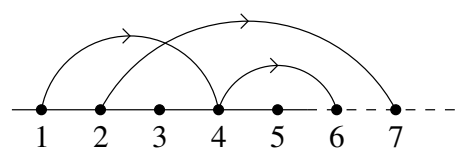

Repeating this for all intervals of length 5 gives

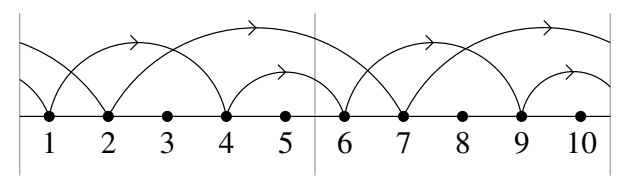

For a given juggling sequence, it is often possible to further decompose into shorter juggling sequences, called primitive juggling sequences, which themselves cannot be further decomposed. These primitive juggling sequences act as basic building blocks for juggling sequences [2]. However, in the other direction, it is not always possible to combine primitive juggling sequences into a longer juggling sequence. Nevertheless, if primitive juggling sequences share a common state (which one can think of as a landing schedule), then we can combine them to form a longer and more complicated juggling sequences. In [2] primitive juggling sequences associated with a specified state are enumerated. Here we mention the related fact concerning $\mathcal{B}_{n, k}$ :

There is a bijection mapping permutations in $\mathcal{B}_{n, k}$ to primitive juggling sequences of period $n$ with $k$ balls that all share a certain state, called the ground state.

The bijection maps $\pi$ to $\phi(\pi)=\left(t_{1}, \ldots, t_{n}\right)$ with $t_{i}=k-i+\pi_{i}$. As a consequence of the above fact and Lemma 1, we can use bubble sort to transform a juggling sequence using $k$ balls to a juggling sequence using $k-1$ balls.

To make this more precise, let $T=\left(t_{1}, \ldots, t_{n}\right)$ be a juggling sequence that corresponds to $\pi \in \mathcal{B}_{n, k}$, and suppose that $T^{\prime}=\left(s_{1}, \ldots, s_{n}\right)$ is the juggling sequence that corresponds to bubble $(\pi)$. Assume that the ball $B$ thrown at time $j$ is the one that lands latest out of all the $n$ throws. In other words, $t_{j}+j$ is the largest element in $\left\{t_{i}+i\right\}_{i=1}^{n}$. Now, write $T=L t_{j} R$ where $L=\left(t_{1}, \ldots, t_{j-1}\right)$ and $R=\left(t_{j+1}, \ldots, t_{n}\right)$. Then we have

$$
T^{\prime}=f_{k}(T)=f_{k}(L) R s,
$$

where $s=t_{j}+j-(n+1)$. In other words, we have removed the ball $B$ thrown at time $j$ and thus throw all balls after time $j$ one time unit sooner. Then at time $n$ we throw the ball B so that it lands one time unit sooner than it would have originally landed. Then we repeat this procedure to all the balls thrown before time $j$.

\section{The polynomials $B_{n, k}(y)$}

In this section we will characterise the polynomials $B_{n, k}(y)$. This is done by first finding a recurrence for the polynomials and then solving the recurrence by exploiting some aspects of their associated char- 
acteristic polynomials. The latter step is quite involved and so we present the special case dealing with $B_{n, 4}(y)$ first.

\subsection{Deriving the recurrence for $B_{n, k}(y)$}

We will derive the following recurrence for $B_{n, k}(y)$.

Theorem 1 For $n \geq 0$,

$$
B_{n+k+1, k}(y)=\sum_{i=1}^{k+1}\left(\begin{array}{c}
k+1 \\
i
\end{array}\right)(y-1)^{i-1} B_{n+k+1-i, k}(y)
$$

with the initial conditions

$$
B_{i, k}(y)=A_{i}(y), \quad 0 \leq i \leq k .
$$

We use the notation $[a, b]=\{i \in \mathbb{Z}: a \leq i \leq b\}$ and $[b]=[1, b]$. Let $A=\left\{a_{1}, \ldots, a_{n}\right\}$ with $a_{1}<\cdots<a_{n}$ be any finite subset of $\mathbb{N}$. The standardization of a permutation $\pi$ on $A$ is the permutation $\operatorname{st}(\pi)$ on $[n]$ obtained from $\pi$ by replacing the integer $a_{i}$ with the integer $i$. Thus $\pi$ and $\operatorname{st}(\pi)$ are order isomorphic. For example, st $(19452)=15342$. If the set $A$ is fixed, the inverse of the standardization map is well defined, and we denote it by $\operatorname{st}_{A}^{-1}(\sigma)$; for instance, with $A=\{1,2,4,5,9\}$, we have $\mathrm{st}_{A}^{-1}(15342)=19452$. Note that st and st ${ }_{A}^{-1}$ each preserve the descent set.

For any set $S \subseteq[n-1]$ we define $\mathcal{A}_{n, k}(S)=\left\{\pi \in \mathcal{B}_{n, k}: \operatorname{Des}(\pi) \supseteq S\right\}$ and

$$
t_{n}(S)=\max \{i \in \mathbb{N}:[n-i, n-1] \subseteq S\} .
$$

Note that $t_{n}(S)=0$ in the case that $n-1$ is not a member of $S$. Now, for any permutation $\pi=\pi_{1} \ldots \pi_{n}$ in $\mathcal{A}_{n, k}(S)$ define

$$
f(\pi)=(\sigma, X), \text { where } \sigma=\operatorname{st}\left(\pi_{1} \ldots \pi_{n-i-1}\right), X=\left\{\pi_{n-i}, \ldots, \pi_{n}\right\} \text { and } i=t_{n}(S) .
$$

Example 1 Let $S=\{3,7,8\}$, and choose the permutation $\pi=138425976$ in $\mathcal{A}_{9,3}(S)$. Notice that $\operatorname{Des}(\pi)=\{3,4,7,8\} \supset$ S. Now $t_{9}(S)=2$. This gives $f(\pi)=(\sigma, X)$ where $\sigma=\operatorname{st}(138425)=136425$ and $X=\left\{\pi_{7}, \pi_{8}, \pi_{9}\right\}=\{6,7,9\}$. Hence $f(138425976)=(136425,\{6,7,9\})$.

Lemma 2 For any $\pi$ in $\mathcal{A}_{n, k}(S)$, the image $f(\pi)$ is in the Cartesian product

$$
\mathcal{A}_{n-i-1, k}\left(S \cap\left[n-t_{n}(S)-2\right]\right) \times\left(\begin{array}{c}
{[n-k, n]} \\
t_{n}(S)+1
\end{array}\right),
$$

where $\left(\begin{array}{l}X \\ m\end{array}\right)$ denotes that set of all m-element subsets of the set $X$.

Proof: Given $\pi \in \mathcal{A}_{n, k}(S)$, let $f(\pi)=(\sigma, X)$. Suppose $i=t_{n}(S)$. Then there are descents at positions $n-i, \ldots, n-1$ (this is an empty sequence in case $i=0$ ). Thus

$$
n \geq \pi_{n-i}>\pi_{n-i+1}>\cdots>\pi_{n-1}>\pi_{n} \geq n-k,
$$

where the last inequality follows from the assumption that $\operatorname{maxdrop}(\pi) \leq k$. Hence $X$ is an $(i+1)$ element subset of $[n-k, n]$, as claimed. Clearly $\sigma \in \mathcal{S}_{n-i-1}$. 
Next we shall show that $\sigma$ is in $\mathcal{A}_{n-i-1, k}$. Notice that the entries of $\left(\pi_{1}, \ldots, \pi_{n-i-1}\right)$ that do not change under standardization are those $\pi_{\ell}$ which are less than $\pi_{n}$. Since these values remain unchanged, the values $\ell-\pi_{\ell}$ are also unchanged and are thus at most $k$.

Let $\left(\pi_{a(1)}, \ldots, \pi_{a(m)}\right)$ be the subsequence of values which are greater than $\pi_{n}$. The smallest value that any of these may take after standardization is $\pi_{n} \geq n-k$. So $\sigma_{a(j)} \geq \pi_{n} \geq n-k$ for all $j \in[1, m]$. Thus $a(j)-\sigma_{a(j)} \leq a(j)-(n-k)=k-(n-a(j)) \leq k$ for all $j \in[1, m]$. Therefore $\ell-\sigma_{\ell} \leq k$ for all $\ell \in[1, n-i-1]$ and so $\sigma \in \mathcal{A}_{n-i-1, k}$.

The descent set is preserved under standardization, and consequently $\sigma$ is in $\mathcal{A}_{n-i-1, k}(S \cap[n-i-2])$, as claimed.

We now define a function $g$ which will be shown to be the inverse of $f$. Let $\pi$ be a permutation in $\mathcal{A}_{m, k}(T)$, where $T$ is a subset of $[m-1]$. We will add $i+1$ elements to $\pi$ to yield a new permutation $\sigma$ in $\mathcal{A}_{m+i, k}(T \cup[m+1, m+i])$. Choose any $(i+1)$-element subset $X$ of the interval $[m+i+1-k, m+i+1]$, and let us write $X=\left\{x_{1}, \ldots, x_{i+1}\right\}$, where $x_{1} \leq \cdots \leq x_{i+1}$. Define

$$
g(\pi, X)=\operatorname{st}_{V}^{-1}\left(\pi_{1} \ldots \pi_{m}\right) x_{i+1} x_{i} \ldots x_{1}, \text { where } V=[m+i+1] \backslash X .
$$

Example 2 Let $T=\{1\}$, and choose the permutation $\pi=3142$ in $\mathcal{A}_{4,3}(T)$. Notice that $\operatorname{Des}(\pi)=$ $\{1,3\} \supseteq T$. Choose $i=2$ and select $a$ subset $X$ from $[4+2+1-3,4+2+1]=\{4,5,6,7\}$ of size $i+1=3$. Let us select $X=\{4,6,7\}$. Now we have $g(\pi, X)=\mathrm{st}_{V}^{-1}(3142) 764=3152764$, where $V$ is the set $[4+2+1] \backslash\{4,6,7\}=\{1,2,3,5\}$.

Lemma 3 If $(\pi, X)$ is in the Cartesian product

$$
\mathcal{A}_{m, k}(T) \times\left(\begin{array}{c}
{[m+i+1-k, m+i+1]} \\
i+1
\end{array}\right)
$$

for some $i>0$ then $g(\pi, X)$ is in

$$
\mathcal{A}_{m+i+1, k}(T \cup[m+1, m+i]) .
$$

Proof: Let $\sigma=g(\pi, X)$. For the first $m$ elements of $\sigma$, since $\sigma_{j} \geq \pi_{j}$ for all $1 \leq j \leq m$, we have $j-\sigma_{j} \leq j-\pi_{j}$ which gives

$$
\max \left\{j-\sigma_{j}: j \in[m]\right\} \leq \max \left\{j-\pi_{j}: j \in[m]\right\} \leq k .
$$

The final $i+1$ elements of $\sigma$ are decreasing so the maxdrop of these elements will be the maxdrop of the final element,

$$
m+i+1-\sigma_{m+i+1}=m+i+1-x_{1} \leq m+i+1-(m+i+1-k)=k .
$$

Thus maxdrop $(\sigma) \leq k$ and so $\sigma \in \mathcal{B}_{m+i+1, k}$. The descents of $\sigma$ will be in the set $T \cup[m+1, m+i]$ since descents are preserved under standardization and the final $i+1$ elements of $\sigma$ are listed in decreasing order. Hence $\sigma \in A_{m+i+1, k}(T \cup[m+1, m+i])$, as claimed.

We omit the straightforward, but a bit tedious, proof of the following important Lemma.

Lemma 4 The function $f$ is a bijection, and $g$ is its inverse. 
Corollary 1 Let $a_{n, k}(S)=\left|\mathcal{A}_{n, k}(S)\right|$ and $i=t_{n}(S)$. Then

$$
a_{n, k}(S)=\left(\begin{array}{c}
k+1 \\
i+1
\end{array}\right) a_{n-(i+1), k}(S \cap[1, n-(i+1)]) .
$$

Proposition 1 For all $n \geq 0$,

$$
\mathcal{B}_{n, k}(y+1)=\sum_{i=1}^{k+1}\left(\begin{array}{c}
k+1 \\
i
\end{array}\right) y^{i-1} \mathcal{B}_{n-i, k}(y+1) .
$$

Proof: Notice that

$$
\begin{aligned}
\mathcal{B}_{n, k}(y+1) & =\sum_{\pi \in \mathcal{B}_{n, k}}(y+1)^{\operatorname{des}(\pi)} \\
& =\sum_{\pi \in \mathcal{B}_{n, k}} \sum_{i=0}^{\operatorname{des}(\pi)}\left(\begin{array}{c}
\operatorname{des}(\pi) \\
i
\end{array}\right) y^{i} \\
& =\sum_{\pi \in \mathcal{B}_{n, k}} \sum_{S \subseteq \operatorname{Des}(\pi)} y^{|S|} \\
& =\sum_{S \subseteq[n-1]} y^{|S|} \sum_{\pi \in \mathcal{A}_{n, k}(S)} 1=\sum_{S \subseteq[n-1]} y^{|S|} a_{n, k}(S) .
\end{aligned}
$$

From Corollary 1 multiply both sides by $y^{|S|}$ and sum over all $S \subseteq[n-1]$. We have

$$
\begin{aligned}
\mathcal{B}_{n, k}(y+1) & =\sum_{S \subseteq[n-1]} y^{|S|}\left(\begin{array}{c}
k+1 \\
t_{n}(S)+1
\end{array}\right) a_{n-\left(t_{n}(S)+1\right), k}\left(S \cap\left[n-\left(t_{n}(S)+2\right)\right]\right) \\
& =\sum_{i \geq 0} \sum_{\substack{S \subseteq[n-1] \\
t n(S)=i}} y^{i} y^{|S|-i}\left(\begin{array}{c}
k+1 \\
i+1
\end{array}\right) a_{n-(i+1), k}(S \cap[n-(i+2)]) \\
& =\sum_{i \geq 0}\left(\begin{array}{c}
k+1 \\
i+1
\end{array}\right) y^{i} \sum_{\substack{S \subseteq[n-1] \\
t_{n}(S)=i}} a_{n-(i+1), k}(S \cap[n-(i+2)]) y^{|S|-i} \\
& =\sum_{i \geq 0}\left(\begin{array}{c}
k+1 \\
i+1
\end{array}\right) y^{i} \sum_{S \subseteq[n-(i+1)]} a_{n-(i+1), k}(S) y^{|S|} \\
& =\sum_{i \geq 0}\left(\begin{array}{c}
k+1 \\
i+1
\end{array}\right) y^{i} \mathcal{B}_{n-(i+1), k}(y+1) \\
& =\sum_{i \geq 1}\left(\begin{array}{c}
k+1 \\
i
\end{array}\right) y^{i-1} \mathcal{B}_{n-i, k}(y+1) .
\end{aligned}
$$


Proof of Theorem 11: Replacing $n$ and $y$ by $n+k+1$ and $y-1$, respectively, in Proposition 1 yields the recurrence (5):

$$
B_{n+k+1, k}(y)=\sum_{i=1}^{k+1}\left(\begin{array}{c}
k+1 \\
i
\end{array}\right)(y-1)^{i-1} B_{n+k+1-i, k}(y)
$$

for $n \geq 0$, with the initial conditions $B_{i, k}(y)=A_{i}(y)$ for $0 \leq i \leq k$.

By multiplying the above recurrence by $z^{n}$ and summing over all $n \geq 0$, we have the generating function $\mathbf{B}_{k}(z, y)$ given in equation (3).

\subsection{Solving the recurrence for $B_{n, 4}(y)$.}

Before we proceed to solve the recurrence for $B_{n, k}$, we first examine the special case of $k=4$ which is quite illuminating. We note that the characteristic polynomial for the recurrence for $B_{n, 4}$ is

$$
h(z)=z^{5}-5 z^{4}+10(1-y) z^{3}-10(1-y)^{2} z^{2}+5(1-y)^{3} z-(1-y)^{4}=\frac{(z-1+y)^{5}-y z^{5}}{1-y} .
$$

Substituting $y=t^{5}$ in the expression above, we see that the roots of $h(z)$ are just

$$
\rho_{j}(t)=\frac{1-t^{5}}{1-\omega^{j} t}, \quad 0 \leq j \leq 4,
$$

where $\omega=\exp \left(\frac{2 \pi \mathfrak{i}}{5}\right)$ is a primitive 5 th root of unity. Hence, the general term for $B_{n, 4}(t)$ can written as

$$
B_{n, 4}(t)=\sum_{i=0}^{4} \alpha_{i}(t) \rho_{i}^{n}(t)
$$

where the $\alpha_{i}(t)$ are appropriately chosen coefficients (polynomials in $t$ ). To determine the $\alpha_{i}(t)$ we need to solve the following system of linear equations:

$$
\sum_{i=0}^{4} \alpha_{i}(t) \rho_{i}^{j}(t)=B_{j, 4}(t)=A_{j}\left(t^{5}\right), \quad 0 \leq j \leq 4 .
$$

Thus, $\alpha_{i}(t)$ can be expressed as the ratio $N_{4, i+1}(t) / D_{4}(t)$ of two determinants. The denominator $D_{4}(t)$ is just a standard Vandermonde determinant whose $(i+1, j+1)$ entry is $\rho_{i}^{j}(t)$. The numerator $N_{4, i+1}(t)$ is formed from $D_{4}(t)$ by replacing the elements $\rho_{i}^{j}(t)$ in the $(i+1)$ st row by $A_{j}\left(t^{5}\right)$. A quick computation (using the symbolic computation package Maple) gives:

$$
\begin{aligned}
D_{4}(t) & =25 \sqrt{5}\left(1-t^{5}\right)^{6} t^{10} \\
N_{4,1}(t) & =5 \sqrt{5}\left(t^{12}+t^{10}+2 t^{9}+4 t^{8}+4 t^{7}+4 t^{5}+4 t^{4}+2 t^{3}+t^{2}+1\right)\left(1-t^{5}\right)^{3}(1-t)^{3} t^{10}
\end{aligned}
$$

and, in general, $N_{4, i+1}(t)=N_{4,1}\left(\omega^{i} t\right)$. Substituting the value $\alpha_{0}(t)=N_{4,1}(t) / D_{4}(t)$ into the first term in the expansion of $B_{n, 4}$, we get

$$
\begin{aligned}
& \alpha_{0}(t)\left(1+t+t^{2}+t^{3}+t^{4}\right)^{n} \\
& \quad=\frac{1}{5}\left(t^{12}+t^{10}+2 t^{9}+4 t^{8}+4 t^{7}+4 t^{5}+4 t^{4}+2 t^{3}+t^{2}+1\right)\left(1+t+t^{2}+t^{3}+t^{4}\right)^{n-3} .
\end{aligned}
$$


Now, since the other four terms $\alpha_{i}(t)\left(1+t+t^{2}+t^{3}+t^{4}\right)^{n}$ arise by replacing $t$ by $\omega^{i} t$ then in the sum of all five terms, the only powers of $t$ that survive are those which have powers which are multiples of 5 . Thus, we can conclude that if we write

$$
\left(t^{12}+t^{10}+2 t^{9}+4 t^{8}+4 t^{7}+4 t^{5}+4 t^{4}+2 t^{3}+t^{2}+1\right)\left(1+t+t^{2}+t^{3}+t^{4}\right)^{n-3}=\sum_{r} \beta(r) t^{r}
$$

then $b_{n, 4}(d)=\beta(5 d)$. In other words, the number of permutations $\pi \in \mathcal{B}_{n, 4}$ with $d$ descents is given by the coefficient of $t^{5 d}$ in the expansion of the above polynomial. Incidentally, the corresponding results for the earlier $\mathcal{B}_{n, i}$ are as follows: $b_{n, 1}(d)=\beta(2 d)$ in the expansion of

$$
(1+t)^{n}=\sum_{r} \beta(r) t^{r}
$$

so $b_{n, 1}(d)=\left(\begin{array}{c}n \\ 2 d\end{array}\right) ; b_{n, 2}(d)=\beta(3 d)$ in the expansion of

$$
\left(1+t^{2}\right)\left(1+t+t^{2}\right)^{n-1}=\sum_{r} \beta(r) t^{r}
$$

and $b_{n, 3}(d)=\beta(4 d)$ in the expansion of

$$
\left(1+t^{2}+2 t^{3}+t^{4}+t^{6}\right)\left(1+t+t^{2}+t^{3}\right)^{n-2}=\sum_{r} \beta(r) t^{r} .
$$

The preceding arguments have now set the stage for dealing with the general case of $B_{n, k}$. Of course, the arguments will be somewhat more involved but it is hoped that treating the above special case will be a useful guide for the reader.

\subsection{Solving the recurrence for $B_{n, k}(y)$}

Theorem 2 We have $B_{n, k}(y)=\sum_{d} \beta_{k}((k+1) d) y^{d}$, where

$$
\sum_{j} \beta_{k}(j) u^{j}=P_{k}(u)\left(1+u+\cdots+u^{k}\right)^{n-k}
$$

and

$$
P_{k}(u)=\sum_{j=0}^{k} A_{k-j}\left(u^{k+1}\right)\left(u^{k+1}-1\right)^{j} \sum_{i=j}^{k}\left(\begin{array}{l}
i \\
j
\end{array}\right) u^{-i} .
$$

The first few values of $P_{k}(u)$ are shown below.

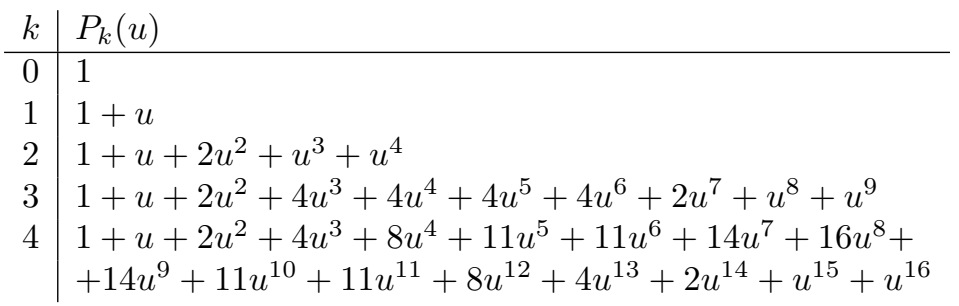

There is clearly a lot of structure in the polynomials $P_{k}(u)$ which will be discussed in the next section. 


\section{The structure of $P_{k}(u)$}

Consider Equation 6 of Theorem 2. We write $P_{k}(u)=\sum_{i=0}^{k^{2}} \alpha_{i} u^{i}$ and define the stretch of $P_{k}(u)$ to be

$$
P P_{k}(u)=\alpha_{0}+\alpha_{k^{2}} u^{k^{2}+k}+\sum_{i=0}^{k} \sum_{j=0}^{k-2} \alpha_{1+i+(k+1) j} u^{2+i+(k+1) j+j} .
$$

What this does to $P_{k}(u)$ is to insert 0 coefficients at every $(k+1)$ st term, starting after $\alpha_{0}$. Thus, the stretched polynomials corresponding to the values of $P_{k}(u)$ given in the array above are:

\begin{tabular}{l|l}
$k$ & $P P_{k}(u)$ \\
\hline 0 & 1 \\
1 & $1+u^{2}$ \\
2 & $1+u^{2}+2 u^{3}+u^{4}+u^{6}$ \\
3 & $1+u^{2}+2 u^{3}+4 u^{4}+4 u^{5}+4 u^{7}+4 u^{8}+2 u^{9}+u^{10}+u^{12}$ \\
4 & $1+u^{2}+2 u^{3}+4 u^{4}+8 u^{5}+11 u^{6}+11 u^{8}+14 u^{9}+16 u^{10}+$ \\
& $+14 u^{11}+11 u^{12}+11 u^{14}+8 u^{15}+4 u^{16}+2 u^{17}+u^{18}+u^{20}$
\end{tabular}

Note that if $P_{k}(u)$ has degree $k^{2}$ then $P P_{k}(u)$ has degree $k^{2}+k$.

Theorem 3 For all $k \geq 1$,

$$
P_{k+1}(u)=P P_{k}(u) \cdot\left(1+u+u^{2}+\cdots+u^{k+1}\right) .
$$

Theorem 4 The coefficients of $P_{k}(u)$ are symmetric and unimodal.

Proof: It follows from Theorem 3 that we can construct the coefficient sequence for $P_{k+1}(u)$ from that of $P_{k}(u)$ by the following rule (where we assume that all coefficients of $u^{t}$ in $P_{k}(u)$ are 0 if $t<0$ or $\left.t>k^{2}\right)$. Namely, suppose we write $P_{k}(u)=\sum_{i=0}^{k^{2}} \alpha_{i} u^{i}$ so that we have the coefficient sequence $A_{k}=\left(\alpha_{0}, \alpha_{1}, \ldots, \alpha_{k^{2}}\right)$. Now form the new sequence $B_{k}=\left(\beta_{0}, \beta_{1}, \ldots \beta_{k^{2}+k}\right)$ by the rule

$$
\beta_{i}=\sum_{j=i-k}^{i} \alpha_{j}, \quad 0 \leq i \leq k^{2}+k .
$$

Finally, starting with $\beta_{0}$, insert duplicate values for the coefficients

$$
\beta_{0}, \beta_{k+1}, \beta_{2(k+1)}, \ldots, \beta_{t(k+1)}, \ldots, \beta_{(k-1)(k+1)} \text { and } \beta_{k(k+1)} .
$$

Thus, this will generate the sequence

$$
\left(\beta_{0}, \beta_{0}, \beta_{1}, \beta_{2}, \ldots, \beta_{k}, \beta_{k+1}, \beta_{k+1}, \beta_{k+2}, \ldots, \beta_{k^{2}+k-1}, \beta_{k^{2}+k}, \beta_{k^{2}+k}\right) .
$$

This new sequence will in fact just be the coefficient sequence $A_{k+1}$ for $P_{k+1}(u)$. For example, starting with $P_{1}(u)=1+u$, we have $A_{1}=(1,1)$ and so $B_{1}=(1,2,1)$. Now, inserting the duplicate values for $\beta_{0}=1$ and $\beta_{2}=1$, we get the coefficient sequence $A_{2}=(1, \mathbf{1}, 2,1, \mathbf{1})$ for $P_{2}(u)=$ $1+u+2 u^{2}+u^{3}+u^{4}$. Repeating this process for $A_{2}$, we sum blocks of length 3 to get $B_{2}=$ $(1,2,4,4,4,2,1)$. Inserting duplicates for entries at positions 0,3 and 6 gives us the new coefficient sequence $A_{3}=(1, \mathbf{1}, 2,4,4, \mathbf{4}, 4,2,1, \mathbf{1})$ of $P_{3}=1+u+2 u^{2}+4 u^{3}+4 u^{4}+4 u^{5}+4 u^{6}+2 u^{7}+u^{8}+u^{9}$, etc. It is also clear from this procedure that if $A_{k}$ is symmetric and unimodal, then so is $B_{k}$, and consequently, so is $A_{k+1}$. This is what we claimed. 


\subsection{An Eulerian identity}

Note that since $P_{k}(u)$ is symmetric and has degree $u^{k^{2}}$, we have $P_{k}(u)=u^{k^{2}} P_{k}\left(\frac{1}{u}\right)$. Replacing $P_{k}(u)$ by its expression in 6, we obtain (with some calculation) the interesting identity

$$
\sum_{j=0}^{a+b}(-1)^{j}\left(\begin{array}{l}
a \\
j
\end{array}\right)(1-x)^{j} A_{a+b-j}(x)=x \sum_{j=0}^{a+b}\left(\begin{array}{l}
b \\
j
\end{array}\right)(1-x)^{j} A_{a+b-j}(x)+\left(\begin{array}{c}
b \\
a+b
\end{array}\right)(1-x)^{a+b+1}
$$

for all integers $a$ and $b$ provided that $a+b \geq 0$.

\section{References}

[1] J. Buhler, D. Eisenberg, R. Graham and C. Wright, Juggling drops and descents, Amer. Math. Monthly 101 (1994), 507-519.

[2] F. Chung, and R. L. Graham, Primitive juggling sequences, Amer. Math. Monthly 115 (2008), 185194.

[3] L. Euler, Methodus universalis series summandi ulterius promota, Commentarii academiae scientiarum imperialis Petropolitanae 8 (1736), 147-158. Reprinted in his Pera Omnia, series 1, volume $14,124-137$.

[4] D. Foata and G. Han, $q$-series in Combinatorics; permutation statistics (Lecture Notes), preliminary edition, 2004.

[5] R. L. Graham, D. E. Knuth and O. Patashnik, Concrete Mathematics, Addison-Wesley, 1994.

[6] D. E. Knuth: The Art of Computer Programming, Vol. 1, Fundamental algorithms. Addison-Wesley, Reading, 1969.

[7] D. E. Knuth, The Art of Computer Programming, Vol. 3, Sorting and Searching, Addison-Wesley, Reading, 2nd ed., 1998.

[8] P. A. MacMahon, Combinatory Analysis, 2 volumes, Cambridge University Press, London, 19151916. Reprinted by Chelsea, New York, 1960.

[9] O. Rodrigues, Note sur les inversions, ou dérangements produits dans les permutations, J. de Math. 4 (1839), 236-240.

[10] J. Shareshian and M. L. Wachs, $q$-Eulerian polynomials: excedance number and major index, Electron. Res. Announc. Amer. Math. Soc. 13 (2007), 33-45.

[11] J. Shareshian and M. L. Wachs, Eulerian quasisymmetric functions, preprint 2009. 
